\title{
Optimal Asynchronous Garbage Collection for RDT Checkpointing Protocols*
}

\author{
Rodrigo Schmidt $^{\star} \quad$ Islene C. Garcia Fernando Pedone $^{\dagger} \quad$ Luiz E. Buzato $^{\ddagger}$ \\ `École Polytechnique Fédérale de Lausanne (EPFL), CH-1015 Lausanne, Switzerland \\ ${ }^{\ddagger}$ Instituto de Computação - Unicamp, 13083-970 Campinas - SP, Brazil \\ †Università della Svizzera Italiana (USI), CH-6904 Lugano, Switzerland \\ E-mails: rodrigo.schmidt@epfl.ch, \{islene, buzato\}@ic.unicamp.br, fernando.pedone@unisi.ch
}

\begin{abstract}
Communication-induced checkpointing protocols that ensure rollback-dependency trackability (RDT) guarantee important properties to the recovery system without explicit coordination. However, to the best of our knowledge, there was no garbage collection algorithm for them which did not use some type of process synchronization, like time assumptions or reliable control message exchanges. This paper addresses the problem of garbage collection for RDT checkpointing protocols and presents an optimal solution for the case where coordination is done only by means of timestamps piggybacked in application messages. Our algorithm uses the same timestamps as off-the-shelf RDT protocols and ensures the tight upper bound on the number of uncollected checkpoints for each process during all the system execution.
\end{abstract}

Keywords: garbage collection, distributed checkpointing, rollback-dependency trackability, rollback-recovery.

\section{Introduction}

Checkpointing is a well-known technique used to build fault-tolerant distributed applications based on rollbackrecovery. Briefly, every process periodically saves the application's local state as a checkpoint and when a failure occurs, the distributed computation restarts from its most recent consistent global checkpoint, or recovery line. A global checkpoint is a set composed of one local checkpoint for each process and it is consistent if it includes the sending of every received message $[6,8]$.

Netzer and $\mathrm{Xu}$ [16] have shown that checkpoint dependencies are created by sequences of messages called zigzag

\footnotetext{
* The work presented in this paper has been partially supported by CAPES, Brazil, and by the Hasler Foundation, Switzerland (project \#1899).
}

paths. Two checkpoints can take part in the same consistent global checkpoint if and only if no zigzag path connects them. A zigzag path can be either causal or non-causal depending on whether the receipt of a message always precedes the sending of the next one. Non-causal zigzag paths may connect a checkpoint to itself and preclude it from taking part in any consistent global checkpoint. A checkpoint involved in such a zigzag cycle is called useless.

If checkpoints are taken autonomously by processes (called basic checkpoints) and no coordination exists, they may become useless and a failure could force the application to roll back to a very initial state, a phenomenon known as the domino effect [17]. Communication-induced checkpointing protocols $[8,15,21]$ avoid the domino effect by piggybacking control information in the application messages and having processes take forced checkpoints, besides the basic ones, to break the non-causal zigzag paths that could create useless checkpoints.

Absence of useless checkpoints is the minimal desired property for communication-induced protocols. Another important property is the possibility of tracking checkpoint dependencies on-the-fly during the application execution using a transitive dependency vector, called rollbackdependency trackability (RDT). Besides ensuring that all checkpoints are useful, the RDT property eases the determination of minimum and maximum consistent global checkpoints containing a given set of local checkpoints, and allows decentralized solutions for recovery line calculation, which has been shown to be helpful in many contexts (e.g., software error recovery, causal distributed breakpoints, deadlock recovery and mobile computing [21]). Moreover, the RDT property minimizes the amount of lost work in a distributed rollback when compared to other domino-free properties [1]. Protocols enforcing the rollback-dependency trackability are called $R D T$ checkpointing protocols $[3,10$, $20,21]$. 
The price of autonomy in communication-induced checkpointing protocols is storage space [2]. The absence of explicit coordination makes it difficult to identify obsolete checkpoints, that is, those not necessary for future recoveries. Existent garbage collection algorithms execute as secondary tasks, eliminating all or a subset of the obsolete checkpoints $[5,8,14,22]$. However, all of them rely either on time assumptions or reliable control message exchanges.

This paper addresses the problem of garbage collection where coordination relies only on information propagated in application messages. We call such garbage collection algorithms asynchronous. Amongst our contributions, we present a characterization of obsolete checkpoints for RDT scenarios and a new algorithm for garbage collection on them. Differently from the previous approaches, ours does not rely on time assumptions or control messages. It runs locally to each process and is based only on the timestamps already propagated by the checkpointing protocol, increasing neither the amount of control information piggybacked nor the execution complexity of the checkpointing middleware. Moreover, we prove that our algorithm is optimal in the sense that no more checkpoints can be eliminated without time assumptions or control messages.

The rest of this paper is organized as follows. Section 2 introduces our model and definitions. Section 3 describes the necessary and sufficient conditions for a checkpoint to be obsolete when rollback-dependency trackability holds. In Section 4, we present and analyze in detail our asynchronous garbage collection algorithm for RDT checkpointing protocols. Section 5 discusses related work in the field and Section 6 concludes the paper.

\section{System model and definitions}

A distributed system is composed of a set $\Pi=$ $\left\{p_{1}, p_{2}, \ldots, p_{n}\right\}$ of processes that communicate only by exchanging messages. The system is asynchronous: there are no assumptions about the time it takes for processes to execute and for messages to be exchanged. Moreover, processes do not share a common clock. Although messages cannot be corrupted, they can be lost or delivered out of order. Process $p_{i}$ 's execution is a sequence of events $e_{i}^{0}, e_{i}^{1}, \ldots$ Internal events are related to the local execution of a process (e.g., local checkpoints) and communication events are related to sending and receiving messages.

A process can fail by crash, stopping its execution and losing its volatile state, but it eventually recovers. Its stable storage persists through failures, preserving the stored information. Finally, we do not assume piecewise determinism, and therefore cannot use event logging during recovery [8].

\subsection{Causality and consistency}

Throughout the paper we use the definitions of causal precedence and consistent cuts, presented next.

Definition 1 Causal precedence [13] - Event $e_{a}^{\alpha}$ causally precedes $e_{b}^{\beta}\left(e_{a}^{\alpha} \rightarrow e_{b}^{\beta}\right)$ iff

(i) $a=b \wedge \beta=\alpha+1$; or

(ii) $\exists m \mid e_{a}^{\alpha}=\operatorname{send}(m) \wedge e_{b}^{\beta}=\operatorname{receive}(m)$; or

(iii) $\exists e_{c}^{\gamma} \mid e_{a}^{\alpha} \rightarrow e_{c}^{\gamma} \wedge e_{c}^{\gamma} \rightarrow e_{b}^{\beta}$.

A cut of a distributed computation contains an initial prefix of the sequence of executed events for each process. A consistent cut is left-closed under causal precedence and represents an instant in a distributed computation, as defined below.

Definition 2 Consistent cut [7] - A cut $\mathcal{C}$ is consistent iff $e \in \mathcal{C} \wedge e^{\prime} \rightarrow e \Rightarrow e^{\prime} \in \mathcal{C}$.

\subsection{Checkpointing}

A local checkpoint written on stable storage is a stable checkpoint. We use $s_{i}^{\gamma}$ to represent the $\gamma$-th stable checkpoint taken by process $p_{i}$ and call $\gamma$ its index. Every process $p_{i}$ starts its execution by storing a stable checkpoint $s_{i}^{0}$. This ensures the existence of at least one global recoverable state. The volatile state of a process $p_{i}$ is called a volatile checkpoint and denoted by $v_{i}$. The set of all checkpoints taken by all the processes in a consistent cut and the dependency relation between them created by the exchanged messages (excluding lost and in-transit messages) form a Checkpoint and Communication Pattern (CCP). We use last_s $(i)$ to refer to the index of the last stable checkpoint taken by process $p_{i}$ in a given CCP and denote $s_{i}^{\text {last_s(i) }}$ by $s_{i}^{\text {last }}$ for simplicity. Moreover, we define $c_{i}^{\gamma}$ as a general checkpoint (or simply checkpoint) of a CCP as follows:

$$
c_{i}^{\gamma}= \begin{cases}s_{i}^{\gamma}, & \gamma \leq \text { last_s }(i) \\ v_{i}, & \gamma=\text { last_s }(i)+1 .\end{cases}
$$

A checkpoint interval $I_{i}^{\gamma}$ is the set of events occurred in process $p_{i}$ between checkpoints $c_{i}^{\gamma-1}$ and $c_{i}^{\gamma}$ (including $c_{i}^{\gamma-1}$ but not $c_{i}^{\gamma}$ ). Figure 1 gives an example of CCP and depicts selected examples of the definitions that we have just presented.

Two checkpoints are inconsistent if they are causally related and consistent otherwise. As a result, a global checkpoint is consistent if, and only if, all its checkpoints are pairwise consistent. In Figure 1, $\left\{v_{1}, s_{2}^{1}, s_{3}^{1}\right\}$ is consistent and $\left\{s_{1}^{0}, s_{2}^{1}, s_{3}^{1}\right\}$ is inconsistent, since $s_{1}^{0} \rightarrow s_{2}^{1}$. A consistent global checkpoint always represents a consistent cut.

Two consistent checkpoints are not necessarily part of the same consistent global checkpoint. Checkpoint dependencies are created by sequences of messages called zigzag 


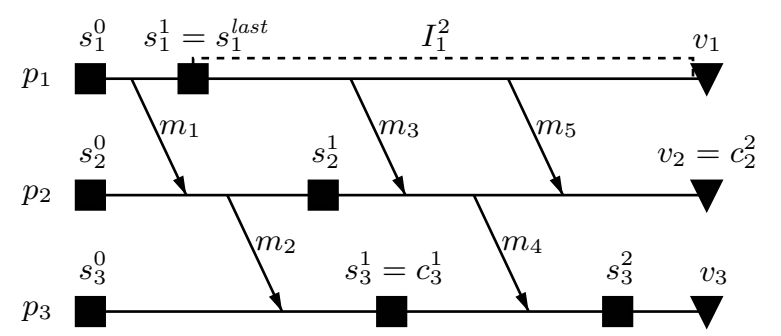

Figure 1. Example of CCP.

paths [16]. We use the relation $c_{a}^{\alpha} \rightsquigarrow c_{b}^{\beta}$ to represent the existence of a zigzag path from $c_{a}^{\alpha}$ to $c_{b}^{\beta}$.

Definition 3 Zigzag path [16] - A sequence of messages $\mu=\left[m_{1}, \ldots, m_{k}\right]$ is a zigzag path which connects $c_{a}^{\alpha}$ to $c_{b}^{\beta}$ iff the conditions below hold:

(i) $p_{a}$ sends $m_{1}$ after $c_{a}^{\alpha}$;

(ii) if $m_{i}, 1 \leq i<k$, is received by $p_{c}$, then $m_{i+1}$ is sent by $p_{c}$ in the same or a later checkpoint interval; and (iii) $p_{b}$ receives $m_{k}$ before $c_{b}^{\beta}$.

A zigzag path can be causal (C-path) or not (Z-path). It is causal if the receipt of each message but the last one causally precedes the send event of the next one in the sequence. In Figure 1, $\left[m_{1}, m_{2}\right]$ and $\left[m_{1}, m_{4}\right]$ are examples of C-paths, and $\left[m_{5}, m_{4}\right]$ is an example of Z-path. A Zpath can connect a checkpoint $c_{i}^{\gamma}$ to itself $\left(c_{i}^{\gamma} \rightsquigarrow c_{i}^{\gamma}\right)$, which renders $c_{i}^{\gamma}$ useless, since it cannot take part in any consistent global checkpoint [16]. Figure 2 illustrates the problem caused by useless checkpoints already mentioned in Section 1. In the scenario we depict, all stable checkpoints but the initial ones are useless (e.g., $\left[m_{2}, m_{1}\right]$ is a Z-path connecting $s_{1}^{1}$ to itself) and, therefore, a single failure would force the entire application to roll back to its initial state, a well-known phenomenon called domino effect [17].

\subsection{Rollback-dependency trackability}

Rollback-dependency trackability is given by the absence of Z-paths which (a) connect a checkpoint to itself or (b) are not doubled by C-paths. This ensures that all check-

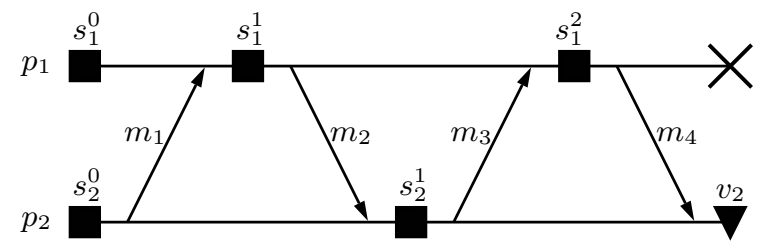

Figure 2. Useless checkpoints and the domino effect. point dependencies are causal and can be tracked by using transitive dependency vectors.

Definition 4 Rollback-dependency trackability [15] - A $C C P$ satisfies rollback-dependency trackability (is RDtrackable) iff for any two checkpoints $c_{i}^{\gamma}$ and $c_{j}^{\iota}, c_{i}^{\gamma} \rightsquigarrow c_{j}^{\iota} \Rightarrow$ $c_{i}^{\gamma} \rightarrow c_{j}^{l}$.

In RD-trackable checkpoint and communication patterns, there are no useless checkpoints, since $c_{i}^{\gamma} \rightsquigarrow c_{i}^{\gamma}$ implies $c_{i}^{\gamma} \rightarrow c_{i}^{\gamma}$, which is impossible. The CCP presented in Figure 1 is RD-trackable. It would not be in the absence of message $m_{3}$ because $\left[m_{5}, m_{4}\right]$ is a Z-path from $s_{1}^{1}$ to $s_{3}^{2}$. Therefore, without $m_{3}$ we would have $s_{1}^{1} \rightsquigarrow s_{3}^{2}$ and $s_{1}^{1} \not s_{3}^{2}$.

RDT checkpointing protocols rely on the model we presented and ensure that the CCP of any consistent cut of the distributed computation is RD-trackable [9]. Therefore, henceforth we assume that all the checkpoint and communication patterns are RD-trackable and we omit this condition in statements of definitions, lemmas and theorems.

\subsection{Rollback-recovery}

The system execution alternates between normal execution periods and recovery sessions, started after some failure. There are many possible approaches to orchestrate recovery sessions $[11,12,14]$. We do not address this problem in the paper and simply assume the existence of a centralized recovery manager which stops the execution of nonfaulty processes, takes their volatile state, calculates and propagates the recovery line, defined below.

Definition 5 Recovery line [22] - Given a CCP and a set offaulty processes $F \subseteq \Pi$, the recovery line $R_{F}$ is the consistent global checkpoint which does not include a volatile checkpoint of a faulty process and minimizes the number of general checkpoints rolled back.

\section{Characterization of obsolete checkpoints}

As execution progresses, new checkpoints are taken and new recovery lines are formed for the possible sets of faulty processes. This makes some stable checkpoints obsolete, allowing the application to discard them in order to save stable storage space.

Definition 6 Obsolete checkpoint - A stable checkpoint is obsolete iff it cannot take part in any future recovery line, even after rollbacks.

Definition 6 is based on the future execution of the distributed application and cannot be used to identify all the obsolete checkpoints in a given CCP. We need a practical 
characterization of obsolete checkpoints and our starting point is recovery line determination. It is known that the recovery line of a faulty set $F$ is unique [22]. The following lemma characterizes it for RD-trackable CCPs. In this extended abstract we omit lemma proofs (they can be found in [18]).

Lemma 1 Given a CCP and a set F offaulty processes, the recovery line $R_{F}$ is determined by:

$$
R_{F}=\bigcup_{i=1}^{n}\left\{c_{i}^{k}, k=\max \left(\gamma \mid \forall p_{f} \in F, s_{f}^{\text {last }} \nrightarrow c_{i}^{\gamma}\right)\right\} .
$$

Informally, the recovery line is composed of the last checkpoint of each process, volatile or not, which is not causally preceded by the last stable checkpoint of any faulty process. Figure 3 gives an example of recovery line determination in a CCP for $F=\left\{p_{2}, p_{3}\right\}$. The gray checkpoints are causally preceded by $s_{2}^{\text {last }}$ or $s_{3}^{\text {last }}$. Thus, by Lemma 1 , the recovery line is composed of the last black checkpoint of each process. Notice that $s_{3}^{\text {last }}$ is not part of the recovery line because it is causally preceded by $s_{2}^{\text {last }}$.

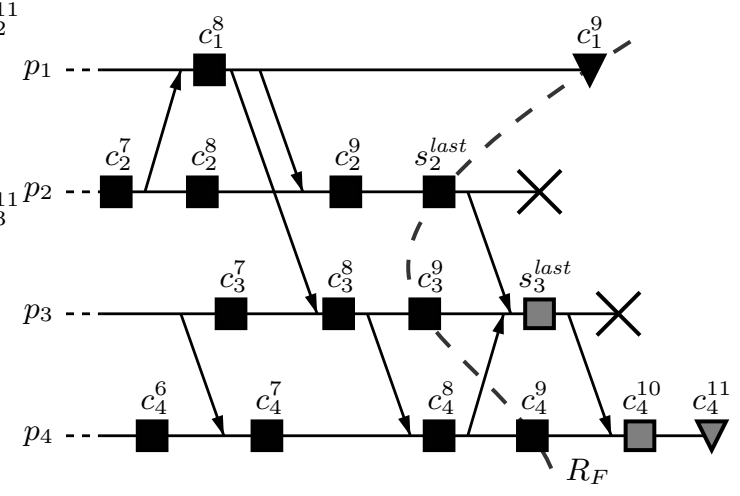

Figure 3. Recovery line determination.

A necessary condition for a checkpoint to be obsolete in a CCP defined by a consistent cut is that it not take part in any of the recovery lines for the $2^{n}$ possible sets of faulty processes (subsets of $\Pi$ ). A checkpoint which does not satisfy this condition in a consistent cut $\mathcal{C}$ is called $\mathcal{C}$-needless.

Definition 7 Needlessness - A stable checkpoint $s_{i}^{\gamma}$ is needless in a consistent cut $\mathcal{C}$ (is $\mathcal{C}$-needless) iff

$$
s_{i}^{\gamma} \in \mathcal{C} \wedge \forall F \subseteq \Pi: s_{i}^{\gamma} \notin R_{F} .
$$

Lemmas 2 and 3 describe, respectively, an easier way to identify needless checkpoints in RD-trackable CCPs and the complete relation between needless and obsolete checkpoints. Similar lemmas have been presented in [22] under different assumptions.
Lemma 2 Every stable checkpoint $s_{i}^{\gamma}$, part of the recovery line for a set of faulty processes $F$ in a CCP, is also part of the recovery line for a single faulty process $p_{f}$ in the same $C C P$, that is,

$$
s_{i}^{\gamma} \in R_{F} \Rightarrow \exists p_{f} \in \Pi \mid s_{i}^{\gamma} \in R_{\left\{p_{f}\right\}} .
$$

Lemma 3 A stable checkpoint $s_{i}^{\gamma}$ is obsolete in the CCP defined by a consistent cut $\mathcal{C}$ iff it is $\mathcal{C}$-needless.

Now we have means to characterize obsolete checkpoints in RD-trackable CCPs using a condition that does not need future knowledge, as we present in Theorem 1.

Theorem 1 Characterization of obsolete checkpoints - A stable checkpoint $s_{i}^{\gamma}$ is obsolete iff there is no process $p_{f}$ such that

$$
s_{f}^{\text {last }} \rightarrow c_{i}^{\gamma+1} \wedge s_{f}^{\text {last }} \nrightarrow s_{i}^{\gamma} .
$$

Proof: By Lemmas 1, 2, 3 and Definition 7.

Theorem 1 says that a process $p_{i}$ must retain the most recent stable checkpoint which is not causally preceded by $s_{f}^{\text {last }}$ for every process $p_{f} \in \Pi$ such that $s_{f}^{\text {last }} \rightarrow v_{i}$. All the other checkpoints of $p_{i}$ are obsolete and may be eliminated. Clearly, the checkpoint $s_{i}^{\text {last }}$ of every process $p_{i}$ is not obsolete because $s_{i}^{\text {last }} \rightarrow v_{i} \wedge s_{i}^{\text {last }} \nrightarrow s_{i}^{\text {last }}$. In Figure 3, for example, there are exactly five obsolete checkpoints: $\left\{c_{2}^{7}, c_{2}^{9}, c_{3}^{8}, c_{4}^{6}, c_{4}^{8}\right\}$.

\section{Asynchronous garbage collection}

Theorem 1 can be used to identify all the existing obsolete checkpoints with a simple algorithm like the one presented by Wang et al. [22]. However, this algorithm is based on reliable control messages exchanged between processes and a central coordinator. Ideally, garbage collection should be as little intrusive as possible, not introducing any overhead in the normal computation. We capture this intuition with the notion of asynchronous garbage collection algorithms, as described next. In this section we also provide such an algorithm and prove its correctness and optimality.

Definition 8 A garbage collection algorithm is asynchronous iff it relies only on information piggybacked in the existent application messages.

\subsection{A suffi cient condition}

We develop next a sufficient condition for asynchronous garbage collection based on causal knowledge only. Let last_ $k_{i}(j)$ denote the index of the last stable checkpoint of process $p_{j}$ known by process $p_{i}$, that is, the last checkpoint of $p_{j}$ which causally precedes the current volatile state of $p_{i}$. If no such stable checkpoint exists, let last_ $k_{i}(j)=-1$. 
For simplicity, we denote $s_{j}^{\text {last_k } k_{i}(j)}$ by $s_{j}^{\text {last } k_{i}}$. Using this terminology, we show in Theorem 2 how to weaken Theorem 1 to get a sufficient condition for garbage collection in RD-trackable CCPs based on causal knowledge.

Theorem 2 A stable checkpoint $s_{i}^{\gamma}$ is obsolete if there is no process $p_{f}$ such that

$$
\text { last_ } k_{i}(f) \geq 0 \wedge s_{f}^{\text {last }_{i}} \rightarrow c_{i}^{\gamma+1} \wedge s_{f}^{\text {last } k_{i}} \nrightarrow s_{i}^{\gamma} .
$$

Proof: Suppose, by contradiction, that $s_{i}^{\gamma}$ satisfies this condition and is not obsolete. By Theorem 1, there is a process $p_{f}$ such that $s_{f}^{\text {last }} \rightarrow c_{i}^{\gamma+1} \wedge s_{f}^{\text {last }} \nrightarrow s_{i}^{\gamma}$. As $s_{f}^{\text {last }} \rightarrow c_{i}^{\gamma+1}$, $p_{i}$ knows $s_{f}^{\text {last }}$ and last_k $k_{i}(f)=$ last_s $(f)$. Therefore, last_ $_{i}(f) \geq 0 \wedge s_{f}^{\text {last }_{i}} \rightarrow c_{i}^{\gamma+1} \wedge s_{f}^{\text {last }_{i}} \nrightarrow s_{i}^{\gamma}$, contradicting our assumptions.

Based on this condition, a process $p_{i}$ could safely retain only its last stable checkpoint that is not causally preceded by $s_{f}^{\text {last } k_{i}}$ for every process $p_{f}$ such that last_ $k_{i}(f) \geq 0$, being sure that all non-obsolete checkpoints are preserved.

\subsection{Dependency vectors}

To implement the condition stated in Theorem 2, we need a dependency tracking mechanism. Dependency vectors [19] capture causal dependencies among checkpoints and are commonly used in RDT checkpointing protocols $[3,10,21]$. In this mechanism, each process $p_{i}$ maintains and propagates inside application messages a size- $n$ dependency vector $D V$, initially $(0, \ldots, 0)$. Entry $D V[i]$ represents the current checkpoint interval of $p_{i}$ and is incremented immediately after a new checkpoint is taken. Every other entry $D V[j], j \neq i$, represents the highest interval index of $p_{j}$ upon which $p_{i}$ depends and is updated every time a message $m$ with a greater value of $m . D V[j]$ arrives at $p_{i}$. When a stable checkpoint is taken, the current dependency vector is stored with it for recovery purposes. We use $D V\left(c_{i}^{\gamma}\right)$ to refer to the dependency vector of checkpoint $c_{i}^{\gamma}$. The following equation derive from the propagation mechanism of dependency vectors [19]:

$$
c_{a}^{\alpha} \rightarrow c_{b}^{\beta} \Longleftrightarrow \alpha<D V\left(c_{b}^{\beta}\right)[a] .
$$

Moreover, as $D V\left(v_{i}\right)[j]$ represents the most recent checkpoint interval from $p_{j}$ known by $p_{i}$, we have that

$$
\text { last_k } k_{i}(j)=D V\left(v_{i}\right)[j]-1 .
$$

Based on it, Corollary 1 restates Theorem 2 in terms of dependency vectors.

Corollary 1 A stable checkpoint $s_{i}^{\gamma}$ is obsolete if there is no process $p_{f}$ such that

$$
D V\left(v_{i}\right)[f]=D V\left(c_{i}^{\gamma+1}\right)[f] \wedge D V\left(v_{i}\right)[f]>D V\left(s_{i}^{\gamma}\right)[f] .
$$

Proof: If we apply Equations 2 and 3 to Theorem 2 we get that $s_{i}^{\gamma}$ is obsolete if there is no process $p_{f}$ such that $D V\left(v_{i}\right)[f]>0 \wedge D V\left(v_{i}\right)[f] \leq D V\left(c_{i}^{\gamma+1}\right)[f] \wedge$ $D V\left(v_{i}\right)[f]>D V\left(s_{i}^{\gamma}\right)[f]$. However, $D V\left(v_{i}\right)[f]>$ $D V\left(s_{i}^{\gamma}\right)[f]$ implies $D V\left(v_{i}\right)[f]>0$ which makes this latter condition superfluous. Moreover, since $D V[i]$ is updated monotonically inside a process, it is impossible to have $D V\left(v_{i}\right)[f]<D V\left(c_{i}^{\gamma+1}\right)[f]$.

Notice that Corollary 1 relies only on values of $D V$ local to process $p_{i}$ and allows it to eliminate obsolete checkpoints without exchanging information with other processes. In the next section we present our complete garbage collection algorithm.

\subsection{Algorithm description}

Our algorithm, named RDT-LGC, simply implements the idea of Theorem 2, identifying obsolete checkpoints as soon as they satisfy the condition of Corollary 1 . We assume that the CCPs created during the execution of the distributed application are always RD-trackable. In Section 4.5 we show how checkpointing and garbage collection could be merged in a single algorithm.

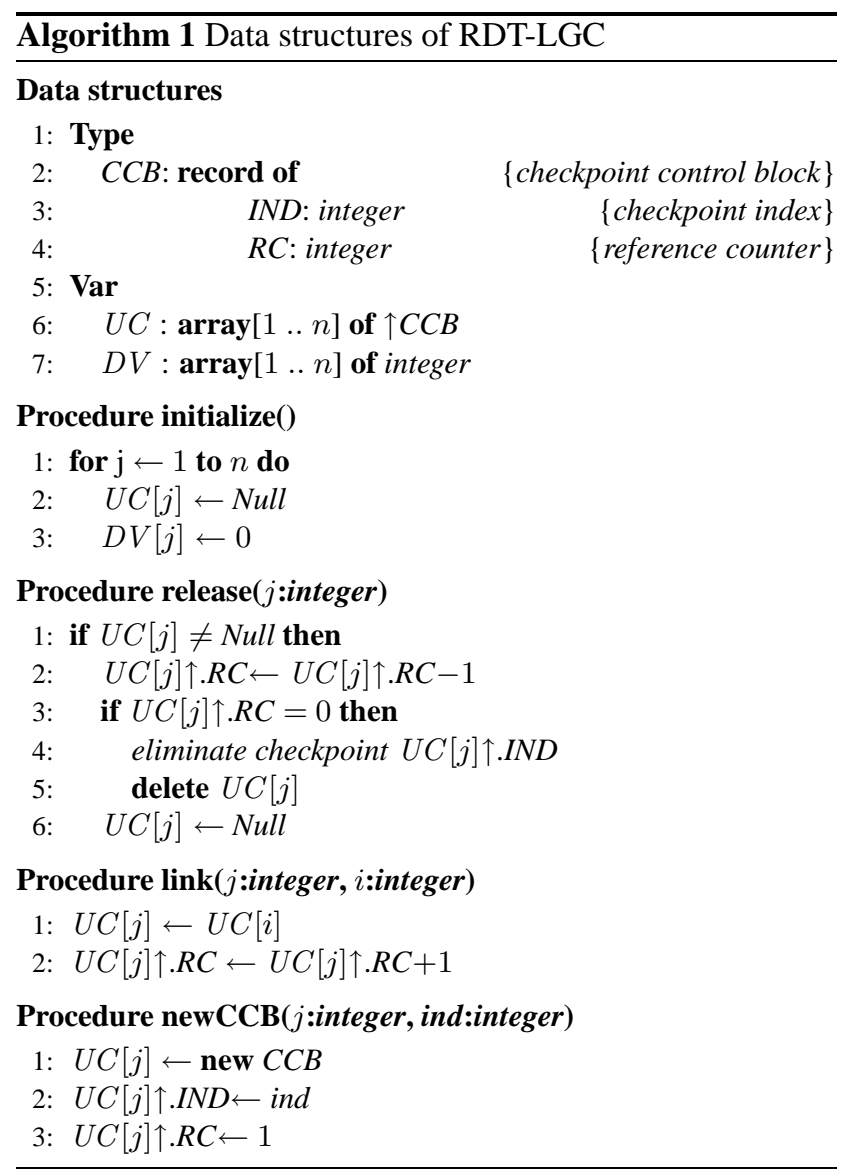




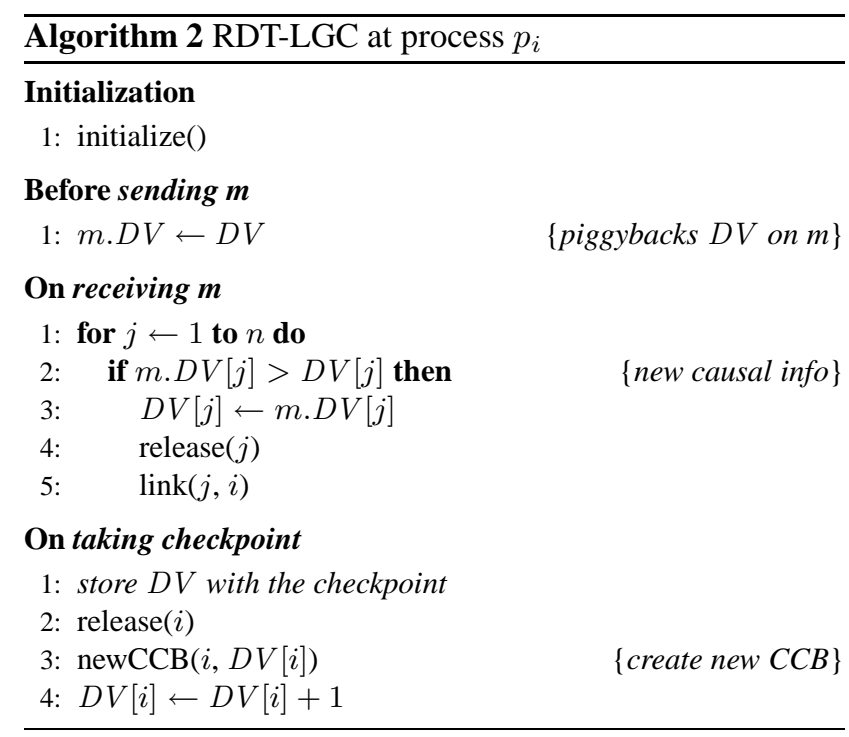

Theorem 2 states that a process $p_{i}$ can retain, for every process $p_{f}$, only the most recent checkpoint not causally preceded by $s_{f}^{\text {last }_{i}}$. Therefore, $p_{i}$ can maintain a simple size- $n$ vector $U C$ (Uncollected Checkpoints) that maps $p_{f}$ to the checkpoint retained because of $p_{f}$. Notice, however, that more than one process can break the condition of Theorem 2 for the same checkpoint of $p_{i}$. Thus, we use a different structure called $C C B$ (Checkpoint Control Block) to represent an uncollected stable checkpoint of $p_{i}$. A $C C B$ keeps track of the checkpoint index and a reference counter storing how many processes deny the checkpoint elimination. $U C$ entries reference $C C B$ s to simplify their update when new causal information is received.

Algorithm 1 presents these data structures, together with the dependency vector, and the basic procedures to manipulate them. Every process has its own instances of the presented data structures. Procedure release decrements the reference counter of the referenced $C C B$ and, if there is no other reference, collects the obsolete checkpoint. Procedure link makes $U C[j]$ reference the same $C C B$ of $U C[i]$. Procedure newCCB creates a new $C C B$ and makes $U C[j]$ reference it. In the following, we explain the RDT-LGC algorithm during normal execution periods and recovery sessions separately.

Normal execution periods. In these periods, RDT-LGC simply updates the data structures mentioned above in order to identify obsolete checkpoints as soon as they satisfy the condition presented in Corollary 1, as shown in Algorithm 2. When a message is received by $p_{i}$ and a new causal dependency from process $p_{j}$ is noticed (line 2), $p_{i}$ must keep track that now, by Theorem $2, p_{j}$ is denying the collection of the last stable checkpoint taken by $p_{i}$. As we show in the sequence, the $C C B$ of this checkpoint is always referenced by $U C[i]$. Therefore, $p_{i}$ updates $D V\left(v_{i}\right)[j]$, re-

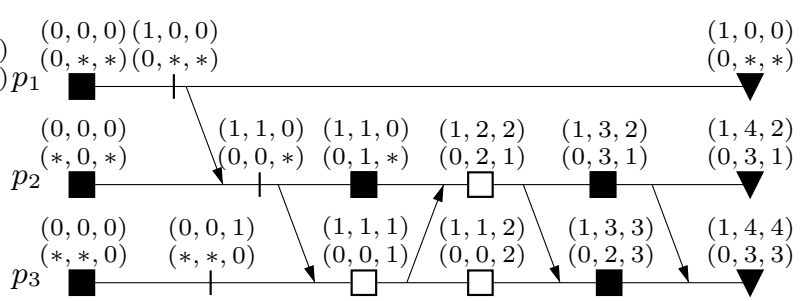

Figure 4. Execution of RDT-LGC.

leases $U C[j]$, and links it to the $C C B$ referenced by $U C[i]$ (lines 3-5). When a new checkpoint is taken, a new $C C B$ is created and $U C[i]$ is updated to reference it (lines 2-3), since $s_{i}^{\text {last }_{i}}=s_{i}^{\text {last }}, s_{i}^{\text {last }} \rightarrow v_{i}$, and $s_{i}^{\text {last }} \nrightarrow s_{i}^{\text {last }}$ (recall Theorem 2). As $p_{i}$ cannot receive new causal information about itself in a message, this is the only way the entry $U C[i]$ is updated, ensuring that it always references the last stable checkpoint of $p_{i}$. The rest of the algorithm refers to dependency vector propagation.

Figure 4 depicts a normal execution of RDT-LGC. For each event shown, we present the contents of $D V$ and $U C$ (in Figure 4, $D V$ is depicted on top of $U C$ ). For simplicity, we show only the checkpoint index of the $C C B$ referenced by an entry $U C[j]$ and represent null references by " $*$ ". Therefore, $U C=(0,0, *)$ means that $U C[0]$ and $U C[1]$ reference the $C C B$ of the first checkpoint taken (index 0), and $U C[2]=$ Null. Remember that $D V[i]$, for a process $p_{i}$, is incremented only after a local checkpoint is taken. By the end of this execution, checkpoints $s_{2}^{2}, s_{3}^{1}$ and $s_{3}^{2}$ (empty squares) have been eliminated. The only obsolete checkpoint not identified by RDT-LGC is $s_{2}^{1}$. It is retained by $p_{2}$ because $p_{2}$ does not know that $p_{3}$ has taken other checkpoints after $s_{3}^{1}$.

Recovery sessions. A simple way to orchestrate a recovery session is through process synchronization $[8,12]$. If global information is available in a single process during recovery, it is possible to eliminate all obsolete checkpoints based on Theorem 1. Let us suppose that every process receives a last interval vector $L I$ such that $L I[j]=$ last $s(j)+1$ in the CCP defined by cut $R_{F}$. This cut represents the global state in which the application starts the following normal execution period. In this context, a process $p_{i}$ that must roll back to a previous checkpoint runs Algorithm 3, where RI indicates the index of the checkpoint to which $p_{i}$ must roll back. Initially, $p_{i}$ eliminates the checkpoints rolled back and calculates the new dependency vector $D V$ (lines 4-6). After that, $p_{i}$ finds for every process $p_{f}$, based on Theorem 1, the stable checkpoint that must be retained by $p_{i}$ because of $p_{f}$ and updates $U C[f]$ accordingly (lines 9-14). Finally, $p_{i}$ eliminates all checkpoints identified as obsolete (lines 15-17). A process $p_{i}$ whose component in $R_{F}$ is its volatile checkpoint does not run this algorithm and can 


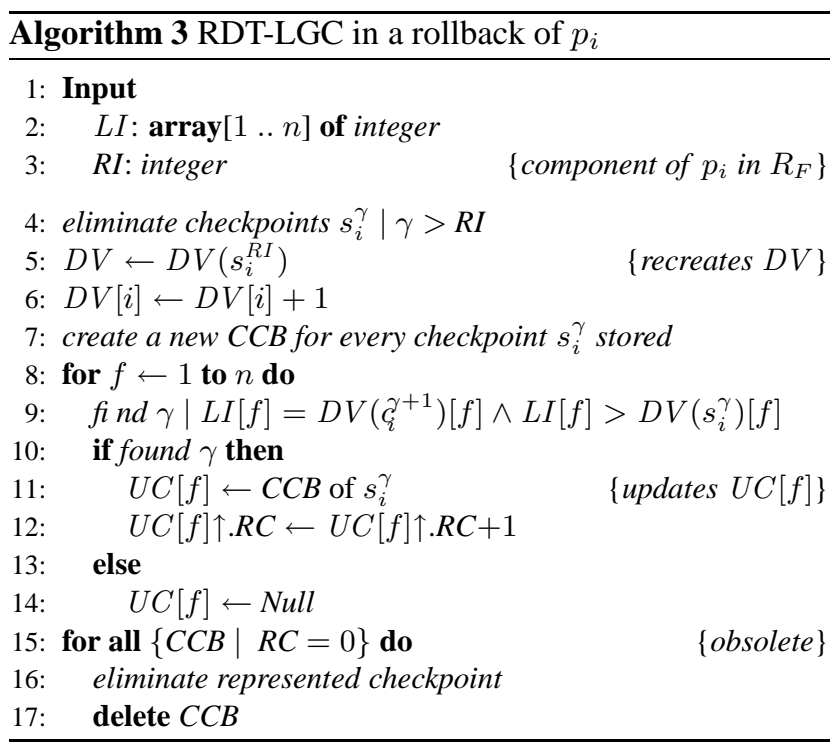

just release any entry $U C[f]$ such that $D V[f]<L I[f]$. If $D V[f]<L I[f]$, the last stable checkpoint of $p_{f}$ does not causally precede $v_{i}$ and, by Theorem 1 , no checkpoint of $p_{i}$ must be retained because of $p_{f}$.

If an uncoordinated algorithm is used for recovery line calculation [21] and no global information is available during the recovery session, a process that must roll back executes Algorithm 3 replacing $L I$ by $D V$ in line 9. This means that the garbage collection will be based on Theorem 2 instead of Theorem 1. A process that does not roll back simply continues its execution, since its volatile state is ensured to be consistent (w.r.t. the recovery line).

\subsection{Correctness and optimality}

RDT-LGC ensures that during the execution, every process $p_{i}$ satisfies the following invariant (see Theorem 3 ). In the following, $U C[f] \equiv s_{i}^{\gamma}$ means that the entry $U C[f]$ references the $C C B$ of $s_{i}^{\gamma}$.

Theorem 3 RDT-LGC satisfies the invariant described by Equation 4 during the execution of every process $p_{i}$ :

$$
s_{f}^{\text {last }} \rightarrow c_{i}^{\gamma+1} \wedge s_{f}^{\text {last }} \nrightarrow s_{i}^{\gamma} \Rightarrow U C[f] \equiv s_{i}^{\gamma} .
$$

Proof: Initially, the invariant is trivially true. Now let us analyze the events where the terms of Equation 4 are modified.

New causal precedence from $p_{f}$ to $p_{i}$. When a new dependency $s_{f}^{\iota} \rightarrow v_{i}$ is created by the receipt of a message, RDT-LGC makes $U C[f]$ reference $s_{i}^{\text {last }}$. If $\iota=$ last_s $(f)$, the new reference makes Equation 4 hold. If $\iota<$ last_s $(f)$, no relation $s_{f}^{\text {last }} \rightarrow c_{i}^{\gamma+1}$ exists and Equation 4 holds independently of $U C[f]$ 's value.
New checkpoint taken by $p_{i}$. When $p_{i}$ takes a new checkpoint, RDT-LGC makes $U C[i]$ reference $s_{i}^{\text {last }}$, since $s_{i}^{\text {last }} \rightarrow v_{i} \wedge s_{i}^{\text {last }} \nrightarrow s_{i}^{\text {last }}$ (left term of Equation 4 when $\left.p_{i}=p_{f}\right)$.

New checkpoint taken by $p_{f}$. When $p_{f} \neq p_{i}$ takes a new checkpoint, the left term of Equation 4 becomes false and the equation as a whole holds independently of $U C[f]$ 's value in $p_{i}$.

Rollback of $p_{i}$. When global information is available during a rollback, Algorithm 3 updates entries $U C[f]$ to satisfy Equation 4 and makes them Null if the left term is false. If there is no global information, Algorithm 3, modified to use $D V$ instead of $L I$, will make this update based on last_k$(f)$ instead of last_s $(f)$. If last_k$k_{i}(f)=$ last_s$_{-}(f)$, the update satisfies the invariant, and if last_k $k_{i}(f)<$ last_s $(f)$, no relation $s_{f}^{\text {last }} \rightarrow$ $c_{i}^{\gamma+1}$ exists and Equation 4 holds independently of $U C[f]$ 's value.

Rollback of $p_{f}$. If $p_{f} \neq p_{i}$ rolls back to a stable checkpoint $s_{f}^{\epsilon}$, this checkpoint becomes $s_{f}^{\text {last }}$ and will not precede any checkpoint of $p_{i}$ in the following normal execution period. Therefore the left term of the equation will be false and the invariant will hold.

From this invariant, we can easily derive a correctness proof for RDT-LGC, as presented in Theorem 4.

Theorem 4 If Equation 4 holds during the execution of every process $p_{i}$, all checkpoints eliminated by RDT-LGC are obsolete.

Proof: By Theorem 1, if the invariant defined by Equation 4 holds, then every non-obsolete checkpoint has at least one entry $U C[j]$ referencing its $C C B$. However, in RDT-LGC, a checkpoint is collected only when there is no entry $U C[j]$ referencing its $C C B$.

We have defined that an asynchronous garbage collection algorithm relies only on causal knowledge and does not exchange control messages. Now we define optimality in this context.

Definition 9 An asynchronous garbage collection algorithm is optimal if it collects all obsolete checkpoints that can be identified using causal knowledge.

Our algorithm is clearly asynchronous, and Theorem 5 shows that it is also optimal.

Theorem 5 RDT-LGC is an optimal asynchronous garbage collection algorithm.

Proof: Suppose, by contradiction, that there is an obsolete checkpoint $s_{i}^{\gamma}$ that can be identified with causal knowledge and is not eliminated by RDT-LGC. As it is not collected 
by RDT-LGC, there is an entry $U C[f]$ in $p_{i}$ that references its $C C B$, which means that $s_{f}^{\text {last }_{i}} \rightarrow c_{i}^{\gamma+1} \wedge s_{f}^{\text {last }_{i}} \not s_{i}^{\gamma}$. However, as it is obsolete and can be identified by causal knowledge, by Theorem $1 p_{i}$ must have known a checkpoint of $p_{f}$ taken after $s_{f}^{\text {last }_{i}}$, contradicting the definition of $s_{f}^{\text {lastk }}$.

\subsection{Analysis and optimizations}

A stable checkpoint $s_{i}^{\gamma}$ is retained by RDT-LGC only if its $C C B$ is referenced by an entry $U C[j]$ in $p_{i}$. Therefore, RDT-LGC retains at most $n$ stable checkpoints in a process during its normal execution, which is the least upper bound on checkpoint space overhead in a single process [22]. Actually, a process may need to store $n+1$ checkpoints for a brief period if lines 1 and 2 of event taking checkpoint in Algorithm 2 cannot be executed atomically. This happens when a process retaining $n$ checkpoints decides to take a new checkpoint, since the last stable checkpoint previously taken will become obsolete only after the new checkpoint is completely stored in stable storage. There are executions where every process reaches this bound, which gives to RDT-LGC a global space overhead of at most $n(n+1)$ stable checkpoints. As an example, consider Figure 5, where the empty squares represent the checkpoints eliminated by RDT-LGC. If, at that moment, all processes decide to take a new checkpoint, a total space of $n(n+1)$ stable checkpoints will be required during the operation. Just after that, $n$ checkpoints will be collected, but $n^{2}$ will remain stored. It is known that when all obsolete checkpoints are identified and eliminated, the global upper bound is $n(n+1) / 2$ checkpoints [22]. However, Theorem 5 shows that it is impossible to give better bounds than RDT-LGC based only on causal knowledge.

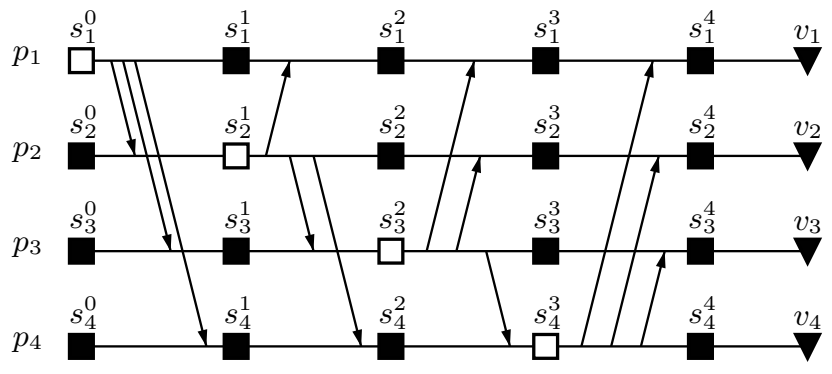

Figure 5. Worst-case scenario for RDT-LGC.

Our algorithm runs locally to each process and relies only on dependency vectors propagated as timestamps in the application messages. Moreover, this task actually drives the time complexity of RDT-LGC in normal execution periods. As efficient RDT checkpointing protocols $[3,10,21]$ also rely on dependency vector propagation, it becomes straightforward to come up with a merged implementation of checkpointing and garbage collection, without increasing the asymptotic time complexity of the former. The only special remark on a merged implementation concerns the treatment of forced checkpoints. As they are triggered by the receipt of a message and supposed to have been taken before its receipt, the implementor must be careful to ensure that forced checkpoints are indeed stored before the execution of the garbage collection related to the receipt of the message. Algorithm 4 shows how RDT-LGC can be integrated in the well-known RDT checkpointing protocol FDAS (Fixed-Dependency-After-Send) [21]. The main difference from a simple implementation of FDAS are the calls to the procedures presented in Algorithm 1, shown in bold.

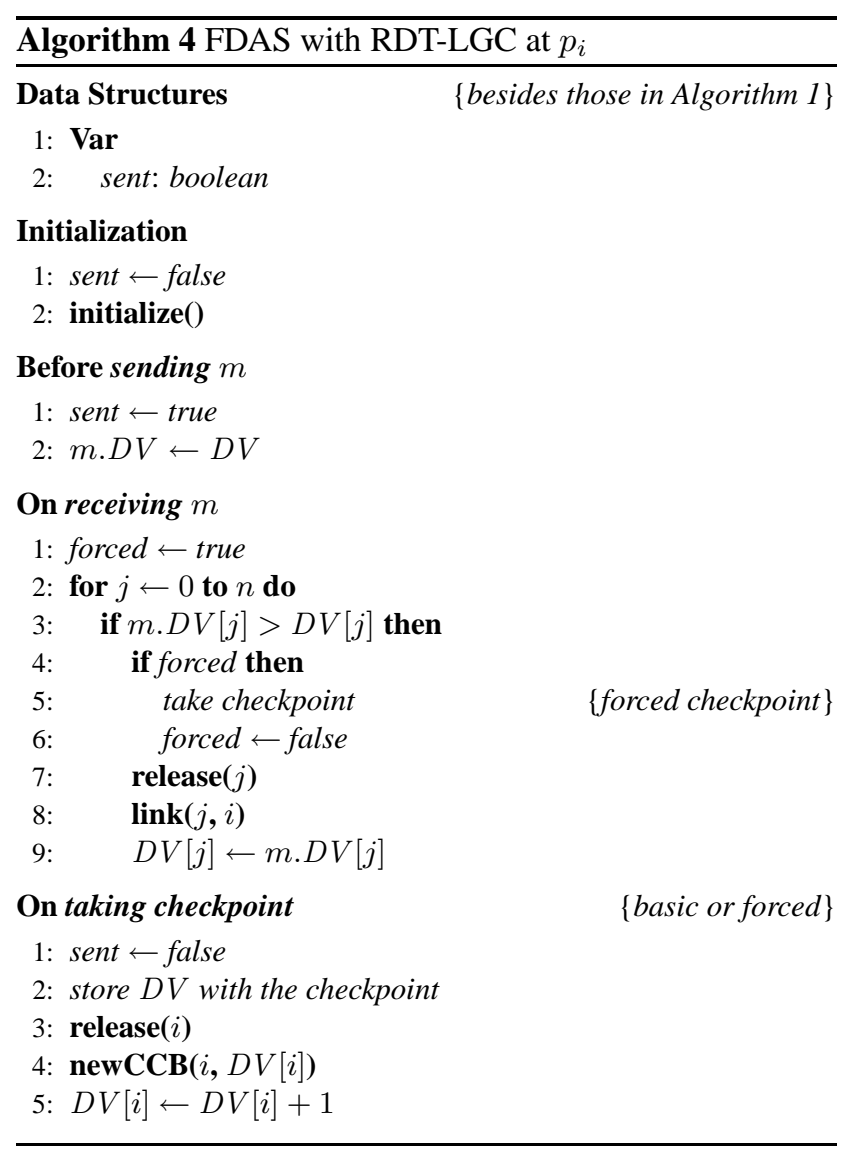

When it comes to time complexity, except for initialize, all procedures in Algorithm 1 execute in $O(1)$ time, which gives an $O(n)$ complexity for all the events shown in Algorithm 2. Algorithm 3 runs in $O(n \log n)$ time if line 9 is implemented using a binary search and $O(n)$ checkpoints are stored. Moreover, for cases in which a process has to roll back without having failed, the algorithm can be improved to a time complexity of $O(n)$ by taking advantage of the existent values in $D V$ and $U C$. 


\section{Related work}

Rollback-dependency trackability was originally presented by Wang [21], who introduced efficient distributed algorithms for calculating minimum and maximum consistent global checkpoints containing a given set of local checkpoints, when the RDT property holds. This seminal work also discusses the application of these algorithms in different scenarios.

RDT can also be defined as the absence of non-causal dependencies, since non-causal zigzag paths must be doubled by a causal relation to ensure on-the-fly trackability $[4,15]$. This observation provided a new perspective on RDT algorithms. Based on it, Agbaria et al. [1] showed that in case of failure, RDT ensures better bounds on the number of rolled back checkpoints than other known domino-free properties.

Much research has been pursued in reducing the number of forced checkpoints in RDT checkpointing protocols. Garcia et al. [10] and Baldoni et al. [3] presented protocols that take fewer forced checkpoints than the protocols presented by Wang [21]. Important results in this context are related to the minimal visible characterization of the RDT property $[4,9]$, which gives the strongest condition to be tested for taking forced checkpoints in order to ensure RDT. Nevertheless, Tsai et al. [20] showed that strong conditions not always translate into a fewer number of forced checkpoints during the whole execution.

Although garbage collection incurs overhead, being an important pragmatic issue in rollback-recovery, it has received little attention in the literature [8]. A simple approach based on the recovery line for the failure of all processes is presented in $[5,8]$. Albeit simple, this algorithm requires process to exchange control messages and does not bound the number of uncollected checkpoints. Wang et al. [22] presented a general characterization of obsolete checkpoints and developed an algorithm that discards all of them and ensures a limit on the number of uncollected checkpoints. However, like the previous approach, it involves the exchange of control messages. The strategy proposed by Manivannan et al. [14] does not involve control message exchanges, but requires processes to take basic checkpoints in known time intervals, which is unfeasible in many practical scenarios.

\section{Concluding remarks}

Garbage collection is highly necessary in systems where the storage space is limited or expensive, like embedded systems and mobile computing. The garbage collection algorithm we presented in this paper ensures that a process will not maintain more than $n$ stored checkpoints during normal execution and does not rely on explicit process synchronization like previous approaches. It relies on the propagation of the same control information as many RDT checkpointing protocols, allowing an efficient merged implementation.

An interesting extension to this work concerns its evaluation in a practical environment. This is motivated by the fact that the theoretical bound on uncollected checkpoints presented in the paper is reached in executions not likely to happen often in practice. Moreover, merged implementations can also be explored in the search for performance improvements. Finally, RDT-LGC is the first garbage collection algorithm based on application messages only. A similar approach could be used to create new efficient garbage collection algorithms based on other properties ensured by checkpointing protocols.

\section{Acknowledgments}

We thank Yi-Min Wang for the explanation about his garbage collection algorithm and the anonymous referees for their valuable comments on the paper.

\section{References}

[1] A. Agbaria, H. Attiya, R. Friedman, and R. Vitenberg. Quantifying Rollback Propagation in Distributed Checkpointing. In Proceedings of the 20th Symposium on Reliable Distributed Systems, pages 36-45, Oct. 2001.

[2] L. Alvisi, E. Elnozahy, S. Rao, S. A. Husain, and A. D. Mel. An Analysis of Communication-Induced Checkpointing. In Proceedings of the 29th IEEE Symposium on Fault-Tolerant Computing, pages 242-249, June 1999.

[3] R. Baldoni, J. M. Hélary, A. Mostéfaoui, and M. Raynal. A Communication-Induced Checkpoint Protocol that Ensures Rollback Dependency Trackability. In Proceedings of the 27th IEEE Symposium on Fault-Tolerant Computing, pages 68-77, June 1997.

[4] R. Baldoni, J. M. Hélary, and M. Raynal. RollbackDependency Trackability: Visible Characterizations. In Proceedings of the 18th ACM Symposium on Principles of Distributed Computing, pages 33-42, May 1999.

[5] B. Bhargava and S. R. Lian. Independent Checkpointing and Concurrent Rollback for Recovery - An Optimistic Approach. In Proceedings of the 7th Symposium on Reliable Distributed Systems, pages 3-12, 1988.

[6] M. Chandy and L. Lamport. Distributed Snapshots: Determining Global States of Distributed Systems. ACM Trans. on Computing Systems, 3(1):63-75, Feb. 1985.

[7] Ö. Babaoğlu and K. Marzullo. Consistent Global States of Distributed Systems: Fundamental Concepts and Mechanisms. In S. Mullender, editor, Distributed Systems, pages 55-96. Addison-Wesley, 1993.

[8] E. N. Elnozahy, L. Alvisi, Y. M. Wang, and D. B. Johnson. A Survey of Rollback-Recovery Protocols in Message-Passing Systems. ACM Computing Surveys, 34(3):375-408, Sept. 2002. 
[9] I. C. Garcia and L. E. Buzato. On the Minimal Characterization of the Rollback-Dependency Trackability Property. In Proceedings of the 21th IEEE Int. Conf. on Distributed Computing Systems, Apr. 2001.

[10] I. C. Garcia and L. E. Buzato. An Effi cient Checkpointing Protocol for the Minimal Characterization of the Operational Rollback-Dependency Trackability. In Proceedings of the 23th IEEE Symposium on Reliable Distributed Systems, Oct. 2004.

[11] Y. Huang and C. Kintala. Software Implemented Fault Tolerance: Technologies and Experiences. In Proceedings of the 16th IEEE Fault-Tolerant Computing Symp., pages 2-9, June 1993.

[12] R. Koo and S. Toueg. Checkpointing and RollbackRecovery for Distributed Systems. IEEE Trans. on Software Engineering, 13:23-31, Jan. 1987.

[13] L. Lamport. Time, Clocks, and the Ordering of Events in a Distributed System. Commun. ACM, 21(7):558-565, July 1978.

[14] D. Manivannan and M. Singhal. A Low-Overhead Recovery Technique Using Quasi-Synchronous Checkpointing. In Proceedings of the 16th IEEE Int. Conf. on Distributed Computing Systems, May 1996.

[15] D. Manivannan and M. Singhal. Quasi-Synchronous Checkpointing: Models, Characterization, and Classifi cation. IEEE Trans. on Parallel and Distributed Systems, 10(7):703-713, July 1999.

[16] R. H. B. Netzer and J. Xu. Necessary and Suffi cient Conditions for Consistent Global Snapshots. IEEE Trans. on Parallel and Distributed Systems, 6(2):165-169, 1995.

[17] B. Randell. System Structure for Software Fault Tolerance. IEEE Trans. on Software Engineering, 1(2):220-232, June 1975.

[18] R. Schmidt, I. C. Garcia, F. Pedone, and L. E. Buzato. Optimal Asynchronous Garbage Collection for Checkpointing Protocols with Rollback-Dependency Trackability. Technical Report IC/2004/45, School of Computer and Communicaiton Sciences, EPFL, 2004.

[19] R. Strom and S. Yemini. Optimistic Recovery in Distributed Systems. ACM Trans. on Computing Systems, 3(3):204-226, Aug. 1985.

[20] J. Tsai, S. Y. Kuo, and Y. M. Wang. Theoretical Analysis for Communication-Induced Checkpointing Protocols with Rollback-Dependency Trackability. IEEE Trans. on Parallel and Distributed Systems, 9(10):963-971, Oct. 1998.

[21] Y. M. Wang. Consistent Global Checkpoints that Contain a Given Set of Local Checkpoints. IEEE Trans. on Computers, 46(4):456-468, Apr. 1997.

[22] Y. M. Wang, P. Y. Chung, I. J. Lin, and W. K. Fuchs. Checkpoint Space Reclamation for Uncoordinated Checkpointing in Message-Passing Systems. IEEE Trans. on Parallel and Distributed Systems, 6(5):546-554, May 1995. 